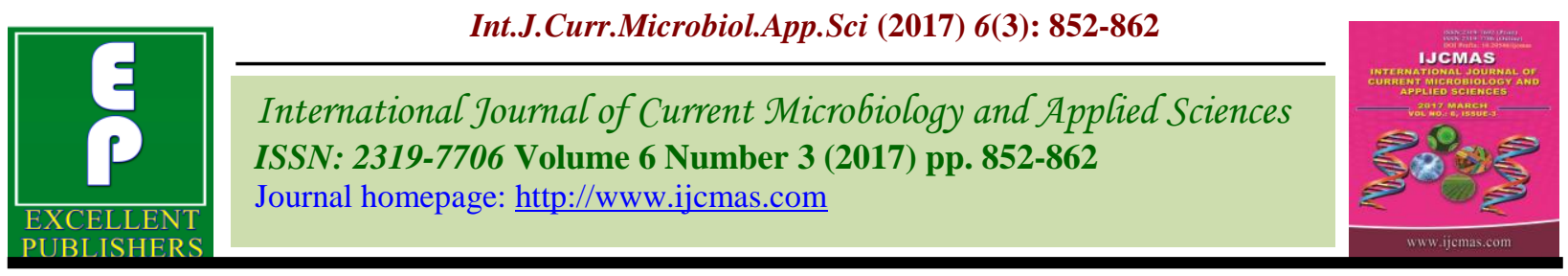

Original Research Article

https://doi.org/10.20546/ijcmas.2017.603.100

\title{
Mapping Soil Nutrient Content Using Geo-statistical Techniques in Kuppam Mandal of A.P, India
}

\author{
P.V.R.M. Reddy*, K.V. Naga Madhuri, T. Giridhara Krishna and V. Nagarjuna \\ Department of Soil Science and Agricultural Chemistry, Institute of Frontier Technology, \\ Regional Agricultural Res. Station, S.V. Agricultural College campus, Tirupati, - 517502 \\ Dist. Chittoor (Andhra Pradesh), India \\ *Corresponding author:
}

\begin{abstract}
Keywords
Geostatistical technique, Kuppam, Precision farming, Soil nutrient analysis, Soil nutrient mapping.

Article Info

Accepted:

15 February 2017

Available Online:

10 March 2017 also. To manage this variation in soil nutrient status precision farming is a technology currently available for sustainable agriculture. This technology enables farm management on the basis of small-scale spatial variability of soil and crop parameters in the field. This study was carried out in Kuppam mandal of Andhra Pradesh. The objective of this study is to determine and map soil nutrient content, both major and micro nutrients variability in Kuppam mandal using geostatistical technique. The major and micronutrients were analyzed and mapped by Geostatistical techniques to quantify the level of spatial nutrient available and predict availability of nutrients at unsampled location also. Results indicated that $99.8 \%$ samples are low and 0.2 sample are medium in available Nitrogen, $13.4 \%$ are low, $19.7 \%$ in medium and $66.7 \%$ are high in available Phosphorus, $47.1 \%$ samples low, $39.8 \%$ medium and $13.1 \%$ samples are high in available Potassium, $78.9 \%$ sample sufficient and $21.1 \%$ samples are deficient in Zinc, $63.6 \%$ samples sufficient and 36.4\% are deficient in Iron, $96.7 \%$ sample sufficient and 3.3\% samples deficient in Copper, 92.8\% samples are sufficient and $7.2 \%$ samples deficient in Manganese. The study revealed the potential and ability of geostatistical techniques in determining and mapping soil nutrient content of study area. Furthermore nutrient maps can be used for balance d fertilization and efficient fertilizer management.
\end{abstract}

\section{A B S T R A C T}

Nutrients are essential for crop growth. Plants absorb nutrients from soil. The quantity and availability of these nutrients varies in various scales, between region, field or within field

\section{Introduction}

Precision agriculture is a practice that has been managed by Remote Sensing (RS) and Geographic Information Systems (GIS) Technology application. This provides the spatial variability more accurately and will be useful to understand and control more precisely what happens on the farm (Mc Cauley et al., 1997). Precision farming has become increasingly significant in the agricultural operations for the site-specific management. The management and manipulation of farming operation are vital decision-making process in improving crop productivity where there is a need to ensure efficiency in the management of agriculture. Information on soil properties in crop field is very important and useful for fertilizer requirement and also to the specific management of the crop and soil. The study of physical, Chemical, Physico-chemical 
properties and more precisely availability of major and micro nutrients, is the most important concept in precision farming (Malek et al., 2007).

This study was planned with a general objective to produce a nutrient status thematic map for both major and micro nutrient variability in Kuppam mandal of Andhra Pradesh. The specific objective is to determine and map nutrient content especially Nitrogen, Phosphorus and Potassium (NPK) and micro nutrient variability in the study area. Later, this map will be used for efficient fertilizer management and convergence in to agricultural action plan of Kuppam mandal.

\section{Materials and Methodology}

\section{Soil sampling, processing and storage}

The entire quality of soil testing results and fertilizer recommendation depends upon soil sampling. Each sample collected must be a true representative of the area being sampled. The accuracy and Utility of the results obtained from the laboratory analysis depends on the sampling precision. For achieving this, 1381 samples were collected at the rate of one sample per every 10 hectares of cultivable land in 64 villages with the help of Global Positioning System (GPS) and latitude and longitude were also recorded. Samples were then kept in labeled plastic bags and brought back to the laboratory for further treatment and analyses. The soil samples were air-dried and sieved to pass $2 \mathrm{~mm}$ mesh sieve.

The available nitrogen was determined by alkaline permanganate method outlined by Subbaih and Asija (1956) and the results are expressed in $\mathrm{kg} \mathrm{ha}^{-1}$. The available phosphorus content was determined by extracting the soil with $0.5 \mathrm{~m} \mathrm{NaHCO}_{3}$ (Olsen et al., 1954) and estimated by developing blue colour using ascorbic acid as reductant on colorimeter
(Olsen and Watanabe, 1965). Available potassium in the soils was extracted by neutral normal ammonium acetate and determined by the flame photometer (Jackson, 1973).The available micronutrients viz., Zinc, Copper, Iron and Manganese were determined in the DTPA extract of soil ( $\mathrm{pH}$ 7.3) using Atomic Absorption Spectrophotometer as outlined by Lindsay and Norwell (1978).

Soil variation is spatial variable. Spatial variation has been recognized for many years (Burrough, 1993). Quantification of spatial variability of soil fertility parameters is essential for formulating land management and fertilizer utilization efficiency. Hence, in this study the spatial distribution of soil properties namely $\mathrm{pH}, \mathrm{EC}$, available macro and micro nutrients is assessed. Spatial variability maps were prepared using interpolation method, Kriging.

\section{Results and Discussion}

The soil nutrient status thematic map of available nitrogen in the study area is shown in Figure 1. The available $\mathrm{N}$ ranged between 10 and $376 \mathrm{~kg} / \mathrm{ha}$. According to Soil Survey Staff (1997), these ranges could be classified as low and medium. Out of 1381 samples analysed for available nitrogen 1378 samples were recorded low available nitrogen status, which amounts for $99.8 \%$ and only $0.2 \%$ samples (3 samples) are having medium available nitrogen (Table 1). The low content of the total $\mathrm{N}$ in the area were due to denitrification, leaching or volatilization of nitrogen from soil. One more reason for very low available nitrogen, might be due to high slope (Cai et al., 1996). Higher slope usually move away the nitrogen to downward direction. The soils have very low available Nitrogen, the holdings are small and the farmers are resource poor and so the yields are very low (Cann, 1994). If we apply recommended doses of nitrogen fertilizers 
there is a chance to increase the productivity by $15 \%$. The available $\mathrm{P}_{2} \mathrm{O}_{5}$ content has got a very wide range 5.6 to $634 \mathrm{~kg} / \mathrm{ha}$. 185 (13.4\%) sample were recorded low available $\mathrm{P}_{2} \mathrm{O}_{5}$ status, $272(19.7 \%)$ samples recorded medium and a maximum of 924 (66.9\%) samples recorded high for available phosphorus status and presented in Figure 2. The high available phosphorus status is due to the continuous application of phosphatic fertilizers season after season. One more reason is the application of phosphatic fertilizers in top dressings and Phosphorus does not leach easily like $\mathrm{NO}_{3}$ (Chen et al., 1999). In contrast to nitrogen, even after repeated trainings and awareness programmes conducted, farmers are applying the phosphatic fertilizers as top dressing also, which resulted in high $\mathrm{P}_{2} \mathrm{O}_{5}$ content in $66.9 \%$ of samples (Table 1). Here quantity of fertilizer application can be reduced if we go based on soil test values and cost of cultivation can be reduced.

Table.1 N P K status in villages of Kuppam mandal

\begin{tabular}{|c|c|c|c|c|c|c|c|c|c|c|}
\hline \multirow[t]{2}{*}{ Village Name } & \multirow[t]{2}{*}{ Total } & \multicolumn{3}{|c|}{$\mathbf{N}$} & \multicolumn{3}{|c|}{$\mathbf{P}$} & \multicolumn{3}{|c|}{$\mathbf{K}$} \\
\hline & & $\mathbf{L}$ & $\mathbf{M}$ & $\mathbf{H}$ & $\mathbf{L}$ & $\mathbf{M}$ & $\mathbf{H}$ & $\mathbf{L}$ & $\mathbf{M}$ & $\mathbf{H}$ \\
\hline Dasegownuru & 4 & 4 & - & - & - & - & 4 & 3 & 1 & - \\
\hline Guttapallecolony & 3 & 3 & - & - & - & 1 & 2 & 3 & - & - \\
\hline Boggupalle & 3 & 3 & - & - & - & - & 3 & - & 3 & - \\
\hline Bairappakottala & 2 & 2 & - & - & - & 1 & 1 & 1 & 1 & - \\
\hline Bandasettipalle & 14 & 14 & - & - & 2 & 1 & 11 & 8 & 5 & 1 \\
\hline Seegalapalle & 11 & 11 & - & - & - & - & 11 & 10 & 1 & - \\
\hline Jarugu & 13 & 13 & - & - & - & 3 & 10 & 10 & 3 & - \\
\hline Parrakuntlapalle & 13 & 13 & - & - & - & - & 13 & 7 & 4 & 2 \\
\hline Challarlapalle & 6 & 6 & - & - & - & 2 & 4 & 3 & 3 & - \\
\hline Yanamanasinapalle & 9 & 9 & - & - & 1 & - & 8 & 4 & 5 & - \\
\hline Oorunayanapalle & 5 & 5 & - & - & - & - & 5 & 3 & 2 & - \\
\hline Oorunayanikothuru & 4 & 4 & - & - & - & 2 & 2 & - & 2 & 2 \\
\hline Gudlanayanipalle & 11 & 11 & - & - & 1 & - & 10 & 6 & 4 & 1 \\
\hline K.d.palle & 8 & 8 & - & - & 2 & 1 & 5 & 3 & 5 & - \\
\hline Yanadipalle & 9 & 9 & - & - & - & 3 & 6 & 6 & 2 & $\mathbf{1}$ \\
\hline Adavibuduguru & 90 & 90 & - & - & 15 & 19 & 56 & 51 & 31 & 8 \\
\hline Kangundi & 42 & 42 & - & - & 6 & 7 & 29 & 22 & 15 & 5 \\
\hline Rajanam & 15 & 15 & - & - & - & 1 & 14 & 5 & 7 & 3 \\
\hline Kangundi bc colony & 29 & 28 & 1 & - & 3 & 1 & 25 & 20 & 7 & 2 \\
\hline Kangundi sc colony & 27 & 27 & - & - & 6 & 7 & 14 & 15 & 8 & 4 \\
\hline Mottakadirinur & 21 & 21 & - & - & - & 4 & 17 & 9 & 10 & 2 \\
\hline Chinnaubba & 20 & 20 & - & - & 3 & 1 & 16 & 6 & 10 & 4 \\
\hline T.sadumuru & 30 & 30 & - & - & 3 & 7 & 20 & 12 & 16 & 2 \\
\hline Ponnamguru & 85 & 85 & - & - & 19 & 12 & 54 & 61 & 22 & 2 \\
\hline R.village kuppam & 32 & 32 & - & - & 8 & 5 & 19 & 20 & 9 & 3 \\
\hline Paipalem & 65 & 65 & - & - & 4 & 4 & 57 & 29 & 23 & 13 \\
\hline
\end{tabular}




\begin{tabular}{|c|c|c|c|c|c|c|c|c|c|c|}
\hline Vasanadu & 48 & 48 & - & - & 3 & 3 & 42 & 14 & 23 & 11 \\
\hline Nadumurur & 20 & 20 & - & - & 4 & 1 & 15 & 5 & 10 & 5 \\
\hline Vendugampalle & 15 & 15 & - & - & - & - & 15 & 5 & 5 & 5 \\
\hline Noolukunta & 26 & 26 & - & - & 1 & 6 & 19 & 11 & 9 & 6 \\
\hline Sajjalapalle & 10 & 10 & - & - & 1 & 3 & 6 & 3 & 5 & 2 \\
\hline Nimmakampalle & 5 & 5 & - & - & 1 & 1 & 3 & - & 3 & 2 \\
\hline Kothapalle & 11 & 11 & - & - & - & 3 & 8 & 4 & 5 & 2 \\
\hline Beyanapalle & 17 & 17 & - & - & 2 & 6 & 9 & 8 & 8 & 1 \\
\hline Pallarlapalle & 27 & 27 & - & - & 7 & 13 & 7 & 9 & 11 & 7 \\
\hline Gonuguru & 21 & 21 & - & - & 3 & 5 & 13 & 1 & 12 & 8 \\
\hline Kakimadugu & 24 & 23 & 1 & - & 2 & 8 & 14 & 3 & 15 & 6 \\
\hline Kunjegownuru & 21 & 20 & 1 & - & 4 & 8 & 9 & 10 & 9 & 2 \\
\hline Mattapalle & 12 & 12 & - & - & 1 & - & 11 & 4 & \multicolumn{2}{|c|}{$r_{\text {Contd }}$} \\
\hline Urlaobanapalle & 27 & 27 & - & - & 1 & 2 & 24 & 9 & $1+$ & + \\
\hline Akararallapalle & 35 & 35 & - & - & 2 & 7 & 26 & 15 & 16 & 4 \\
\hline Kathimanupalle & 7 & 7 & - & - & - & - & 7 & 1 & 3 & 3 \\
\hline Chinnabangarunatham & 7 & 7 & - & - & 3 & 1 & 3 & 5 & 1 & 1 \\
\hline Peddabangarunatham & 18 & 18 & - & - & 4 & 5 & 9 & 6 & 11 & 1 \\
\hline Avulanatham & 17 & 17 & - & - & 1 & 4 & 12 & 2 & 11 & 4 \\
\hline Chikkunatham & 20 & 20 & - & - & 5 & 2 & 13 & 8 & 12 & - \\
\hline Peddagopannapalle & 9 & 9 & - & - & - & 5 & 4 & 2 & 5 & 2 \\
\hline Adavimulakalapalle & 23 & 23 & - & - & 4 & 2 & 17 & 9 & 8 & 6 \\
\hline Kanumapacharlapalle & 15 & 15 & - & - & 4 & 4 & 7 & 10 & 4 & 1 \\
\hline Vasanadu gollapalle & 7 & 7 & - & - & - & 2 & 5 & 5 & - & 2 \\
\hline Bodaguttapalle & 9 & 9 & - & - & 2 & 1 & 6 & 4 & 4 & 1 \\
\hline Bairanganapalle & 17 & 17 & - & - & 3 & - & 14 & 9 & 6 & 2 \\
\hline Bandasettipalle & 9 & 9 & - & - & 4 & 2 & 3 & 6 & 2 & 1 \\
\hline Kamathamuru & 8 & 8 & - & - & - & 2 & 6 & 2 & 2 & 4 \\
\hline Illajanuru & 9 & 9 & - & - & - & 2 & 7 & 5 & 4 & - \\
\hline Mulakalapalle & 13 & 13 & - & - & 1 & 2 & 10 & 6 & 3 & 4 \\
\hline V56 & 4 & 4 & - & - & - & - & 4 & 1 & 3 & - \\
\hline Venkatesapuram & 7 & 7 & - & - & - & 4 & 3 & 3 & 4 & - \\
\hline Varamuru & 10 & 10 & - & - & 4 & 3 & 3 & 8 & 2 & - \\
\hline Guttappanayanipalle & 10 & 10 & - & - & 2 & 4 & 4 & 3 & 4 & 3 \\
\hline Marapalle & 7 & 7 & - & - & - & - & 7 & 3 & 2 & 2 \\
\hline Boggupalle & 8 & 8 & - & - & 2 & 3 & 3 & 4 & 4 & - \\
\hline Peddaboggupalle & 14 & 14 & - & - & 3 & 4 & 7 & 8 & 4 & 2 \\
\hline Kuttiganipalle & 8 & 8 & - & - & 1 & - & 7 & 1 & 3 & 4 \\
\hline Chinnakurabalapalle & 8 & 8 & - & - & 1 & - & 7 & 5 & 3 & - \\
\hline Total no. of samples & 1381 & 1378 & 3 & $\mathbf{0}$ & 185 & 272 & 924 & 651 & \begin{tabular}{|l|}
549 \\
\end{tabular} & 181 \\
\hline Percentage & 100 & 99.8 & \begin{tabular}{|l|l|}
0.2 \\
\end{tabular} & 0.0 & 13.4 & 19.7 & 66.9 & 47.1 & \begin{tabular}{|l|}
39.8 \\
\end{tabular} & 13.1 \\
\hline
\end{tabular}


Table.2 Micro nutrient status in villages of Kuppam mandal

\begin{tabular}{|c|c|c|c|c|c|c|c|c|c|}
\hline \multirow[t]{2}{*}{ Village Name } & \multirow[t]{2}{*}{ Total } & \multicolumn{2}{|c|}{$\mathbf{Z n}$} & \multicolumn{2}{|c|}{$\mathbf{F e}$} & \multicolumn{2}{|c|}{$\mathbf{C u}$} & \multicolumn{2}{|c|}{ Mn } \\
\hline & & $\mathbf{S}$ & D & $\mathbf{S}$ & D & $\mathbf{S}$ & $\mathbf{D}$ & $\mathbf{S}$ & $\mathbf{D}$ \\
\hline Dasegownuru & 4 & 4 & - & 2 & 2 & 4 & - & 4 & - \\
\hline Guttapallecolony & 3 & 2 & 1 & 1 & 2 & 3 & - & 3 & - \\
\hline Boggupalle & 3 & 3 & - & 1 & 2 & 3 & - & 3 & - \\
\hline Bairappakottala & 2 & $\mathbf{1}$ & $\mathbf{1}$ & $\mathbf{1}$ & 1 & 2 & - & 1 & 1 \\
\hline Bandasettipalle & 14 & 11 & 3 & 12 & 2 & 14 & - & 13 & 1 \\
\hline Seegalapalle & 11 & 10 & $\mathbf{1}$ & 10 & 1 & 10 & $\mathbf{1}$ & 11 & - \\
\hline Jarugu & 13 & 11 & 2 & 11 & 2 & 13 & - & 13 & - \\
\hline Parrakuntlapalle & 13 & 13 & - & 8 & 5 & 13 & - & 13 & - \\
\hline Challarlapalle & 6 & 5 & 1 & 3 & 3 & 6 & - & 6 & - \\
\hline Yanamanasinapalle & 9 & 8 & $\mathbf{1}$ & 9 & - & 9 & - & 8 & 1 \\
\hline Oorunayanapalle & 5 & $\mathbf{3}$ & 2 & 3 & 2 & 5 & - & 5 & - \\
\hline Oorunayanikothuru & 4 & 3 & $\mathbf{1}$ & 1 & 3 & 4 & - & 4 & - \\
\hline Gudlanayanipalle & 11 & 11 & - & 6 & 5 & 11 & - & 11 & - \\
\hline K.d.palle & 8 & 7 & $\mathbf{1}$ & 2 & 6 & 8 & - & 7 & 1 \\
\hline Yanadipalle & 9 & 6 & 3 & 4 & 5 & 9 & - & 9 & - \\
\hline Adavibuduguru & 90 & 86 & 4 & 68 & 22 & 88 & 2 & 87 & 3 \\
\hline Kangundi & 42 & 42 & - & 35 & 7 & 42 & - & 42 & - \\
\hline Rajanam & 15 & 15 & - & 13 & 2 & 15 & - & 15 & - \\
\hline Kangundi be colony & 29 & 18 & 11 & 21 & 8 & 25 & 4 & 29 & - \\
\hline Kangundi sc colony & 27 & 25 & 2 & 16 & 11 & 23 & 4 & 26 & 1 \\
\hline Mottakadirinur & 21 & 16 & 5 & 16 & 5 & 19 & 2 & 20 & 1 \\
\hline Chinnaubba & 20 & 14 & 6 & 9 & 11 & 20 & - & 19 & 1 \\
\hline T.sadumuru & 30 & 22 & 8 & 11 & 19 & 28 & 2 & 25 & 5 \\
\hline Ponnamguru & 85 & 45 & 40 & 57 & 28 & 81 & 4 & 76 & 9 \\
\hline R.village kuppam & 32 & 21 & 11 & 20 & 12 & 32 & - & 28 & 4 \\
\hline Paipalem & 65 & 62 & 3 & 32 & 33 & 64 & 1 & 61 & 4 \\
\hline Vasanadu & 48 & 42 & 6 & 25 & 23 & 48 & - & 48 & - \\
\hline Nadumurur & 20 & 17 & $\mathbf{3}$ & 8 & 12 & 20 & - & 20 & - \\
\hline Vendugampalle & 15 & 15 & - & 7 & 8 & 15 & - & 11 & 4 \\
\hline Noolukunta & 26 & 23 & $\mathbf{3}$ & 12 & 14 & 24 & 2 & 19 & 7 \\
\hline Sajjalapalle & 10 & 6 & 4 & 6 & 4 & 10 & - & 9 & 1 \\
\hline Nimmakampalle & 5 & 4 & $\mathbf{1}$ & 1 & 4 & 4 & $\mathbf{1}$ & 5 & - \\
\hline Kothapalle & 11 & 9 & 2 & 1 & 10 & 11 & - & 10 & 1 \\
\hline Beyanapalle & 17 & 13 & 4 & 12 & 5 & 17 & - & 17 & - \\
\hline Pallarlapalle & 27 & 17 & 10 & 18 & 9 & 27 & - & 23 & 4 \\
\hline Gonuguru & 21 & 13 & 8 & 3 & 18 & 20 & 1 & 19 & 2 \\
\hline Kakimadugu & 24 & 17 & 7 & 18 & 6 & 24 & - & 19 & 5 \\
\hline
\end{tabular}




\begin{tabular}{|l|c|c|c|c|c|c|c|c|c|}
\hline Kunjegownuru & 21 & $\mathbf{1 6}$ & $\mathbf{5}$ & $\mathbf{1 5}$ & $\mathbf{6}$ & $\mathbf{1 9}$ & $\mathbf{2}$ & $\mathbf{1 9}$ & 2 \\
\hline Mattapalle & 12 & $\mathbf{1 0}$ & $\mathbf{2}$ & $\mathbf{8}$ & $\mathbf{4}$ & $\mathbf{1 2}$ & - & \multicolumn{2}{|c|}{ ontd... } \\
\hline Urlaobanapalle & 27 & $\mathbf{2 4}$ & $\mathbf{3}$ & $\mathbf{1 5}$ & $\mathbf{1 2}$ & $\mathbf{2 6}$ & $\mathbf{1}$ & $\mathbf{2 0}$ & I \\
\hline Akararallapalle & 35 & $\mathbf{3 2}$ & $\mathbf{3}$ & $\mathbf{1 9}$ & $\mathbf{1 6}$ & $\mathbf{3 5}$ & - & $\mathbf{3 4}$ & 1 \\
\hline Kathimanupalle & 7 & $\mathbf{6}$ & $\mathbf{1}$ & $\mathbf{4}$ & $\mathbf{3}$ & $\mathbf{7}$ & - & $\mathbf{6}$ & 1 \\
\hline Chinnabangarunatham & 7 & $\mathbf{2}$ & $\mathbf{5}$ & $\mathbf{4}$ & $\mathbf{3}$ & $\mathbf{7}$ & - & $\mathbf{7}$ & - \\
\hline Peddabangarunatham & 18 & $\mathbf{1 0}$ & $\mathbf{8}$ & $\mathbf{9}$ & $\mathbf{9}$ & $\mathbf{1 8}$ & - & $\mathbf{1 6}$ & 2 \\
\hline Avulanatham & 17 & $\mathbf{1 6}$ & $\mathbf{1}$ & $\mathbf{1 4}$ & $\mathbf{3}$ & $\mathbf{1 7}$ & - & $\mathbf{1 7}$ & - \\
\hline Chikkunatham & 20 & $\mathbf{1 7}$ & $\mathbf{3}$ & $\mathbf{1 4}$ & $\mathbf{6}$ & $\mathbf{2 0}$ & - & $\mathbf{1 9}$ & 1 \\
\hline Peddagopannapalle & 9 & $\mathbf{8}$ & $\mathbf{1}$ & $\mathbf{9}$ & - & $\mathbf{9}$ & - & $\mathbf{7}$ & 2 \\
\hline Adavimulakalapalle & 23 & $\mathbf{1 9}$ & $\mathbf{4}$ & $\mathbf{1 2}$ & $\mathbf{1 1}$ & $\mathbf{2 3}$ & - & $\mathbf{2 3}$ & - \\
\hline Kanumapacharlapalle & 15 & $\mathbf{1 0}$ & $\mathbf{5}$ & $\mathbf{7}$ & $\mathbf{8}$ & $\mathbf{1 5}$ & - & $\mathbf{1 5}$ & - \\
\hline Vasanadu gollapalle & 7 & $\mathbf{7}$ & - & $\mathbf{6}$ & $\mathbf{1}$ & $\mathbf{7}$ & - & $\mathbf{7}$ & - \\
\hline Bodaguttapalle & 9 & $\mathbf{8}$ & $\mathbf{1}$ & $\mathbf{5}$ & $\mathbf{4}$ & $\mathbf{9}$ & - & $\mathbf{9}$ & - \\
\hline Bairanganapalle & 17 & $\mathbf{1 6}$ & $\mathbf{1}$ & $\mathbf{1 0}$ & $\mathbf{7}$ & $\mathbf{1 7}$ & - & $\mathbf{1 5}$ & 2 \\
\hline Bandasettipalle & 9 & $\mathbf{3}$ & $\mathbf{6}$ & $\mathbf{7}$ & $\mathbf{2}$ & $\mathbf{9}$ & - & $\mathbf{8}$ & 1 \\
\hline Kamathamuru & 8 & $\mathbf{3}$ & $\mathbf{5}$ & $\mathbf{6}$ & $\mathbf{2}$ & $\mathbf{8}$ & - & $\mathbf{8}$ & - \\
\hline Illajanuru & 9 & $\mathbf{8}$ & $\mathbf{1}$ & $\mathbf{7}$ & $\mathbf{2}$ & $\mathbf{9}$ & - & $\mathbf{8}$ & 1 \\
\hline Mulakalapalle & 13 & $\mathbf{1 0}$ & $\mathbf{3}$ & $\mathbf{3}$ & $\mathbf{1 0}$ & $\mathbf{1 3}$ & - & $\mathbf{1 3}$ & - \\
\hline V56 & 4 & $\mathbf{3}$ & $\mathbf{1}$ & $\mathbf{4}$ & - & $\mathbf{4}$ & - & $\mathbf{4}$ & - \\
\hline Venkatesapuram & 7 & $\mathbf{2}$ & $\mathbf{5}$ & $\mathbf{5}$ & $\mathbf{2}$ & $\mathbf{7}$ & - & $\mathbf{7}$ & - \\
\hline Varamuru & 10 & $\mathbf{5}$ & $\mathbf{5}$ & $\mathbf{8}$ & $\mathbf{2}$ & $\mathbf{1 0}$ & - & $\mathbf{1 0}$ & - \\
\hline Guttappanayanipalle & 10 & $\mathbf{9}$ & $\mathbf{1}$ & $\mathbf{8}$ & $\mathbf{2}$ & $\mathbf{1 0}$ & - & $\mathbf{1 0}$ & - \\
\hline Marapalle & 7 & $\mathbf{7}$ & - & $\mathbf{3}$ & $\mathbf{4}$ & $\mathbf{7}$ & - & $\mathbf{7}$ & - \\
\hline Boggupalle & 8 & $\mathbf{8}$ & - & $\mathbf{5}$ & $\mathbf{3}$ & $\mathbf{7}$ & $\mathbf{1}$ & $\mathbf{8}$ & - \\
\hline Peddaboggupalle & 14 & $\mathbf{1 4}$ & $\mathbf{-}$ & $\mathbf{8}$ & $\mathbf{6}$ & $\mathbf{1 4}$ & - & $\mathbf{1 2}$ & 2 \\
\hline Kuttiganipalle & 8 & $\mathbf{8}$ & - & $\mathbf{7}$ & $\mathbf{1}$ & $\mathbf{8}$ & - & $\mathbf{8}$ & - \\
\hline Chinnakurabalapalle & 8 & $\mathbf{3}$ & $\mathbf{5}$ & $\mathbf{6}$ & $\mathbf{2}$ & $\mathbf{8}$ & - & $\mathbf{8}$ & - \\
\hline Total no. of samples & 1381 & 1089 & 292 & 878 & 503 & 1335 & 46 & 1282 & 99 \\
\hline Percentage & 100 & 78.9 & 21.1 & 63.6 & 36.4 & 96.7 & 3.3 & 92.8 & 7.2 \\
\hline & & & & & & & & & \\
\hline
\end{tabular}


Fig.1

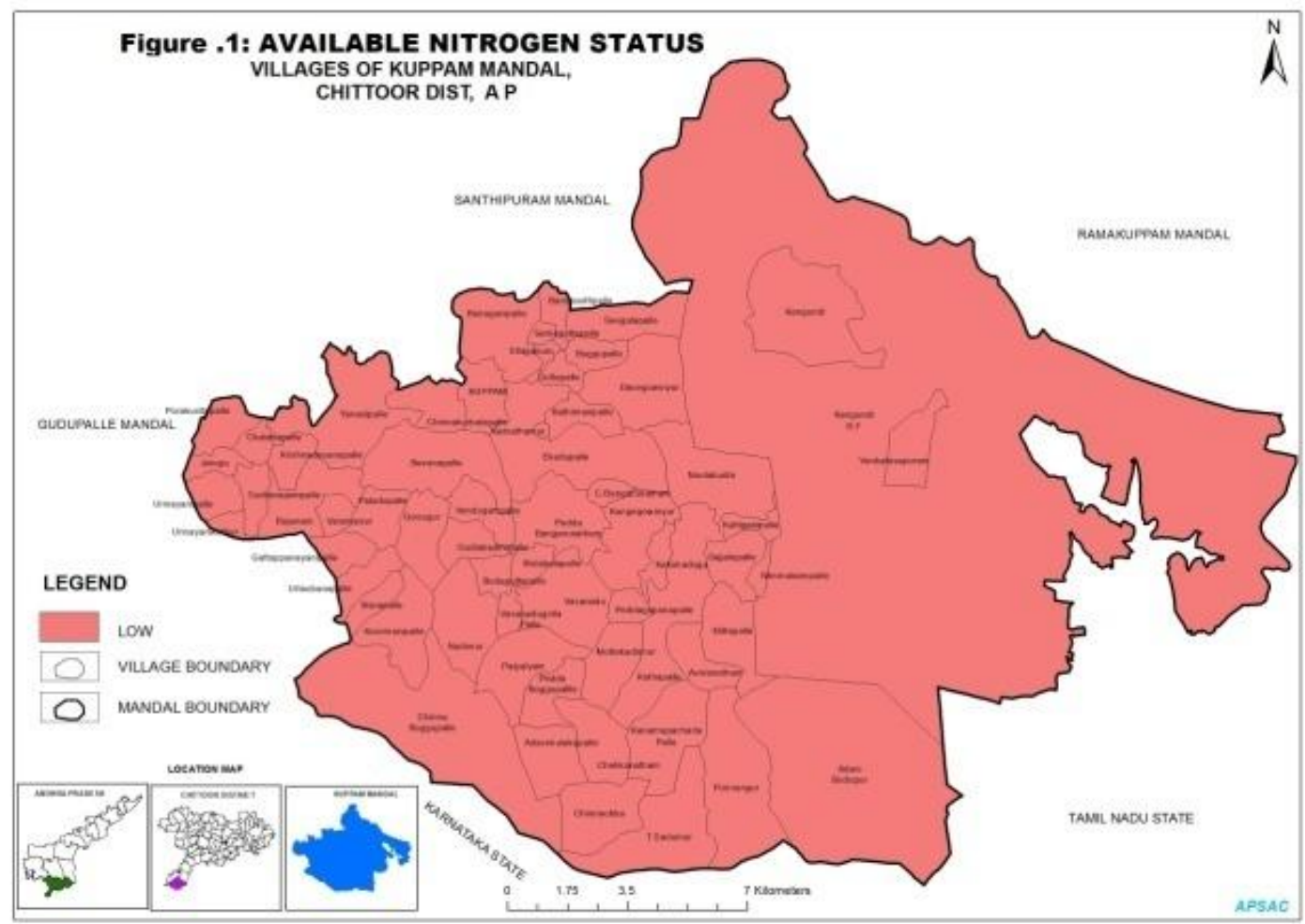

Fig.2

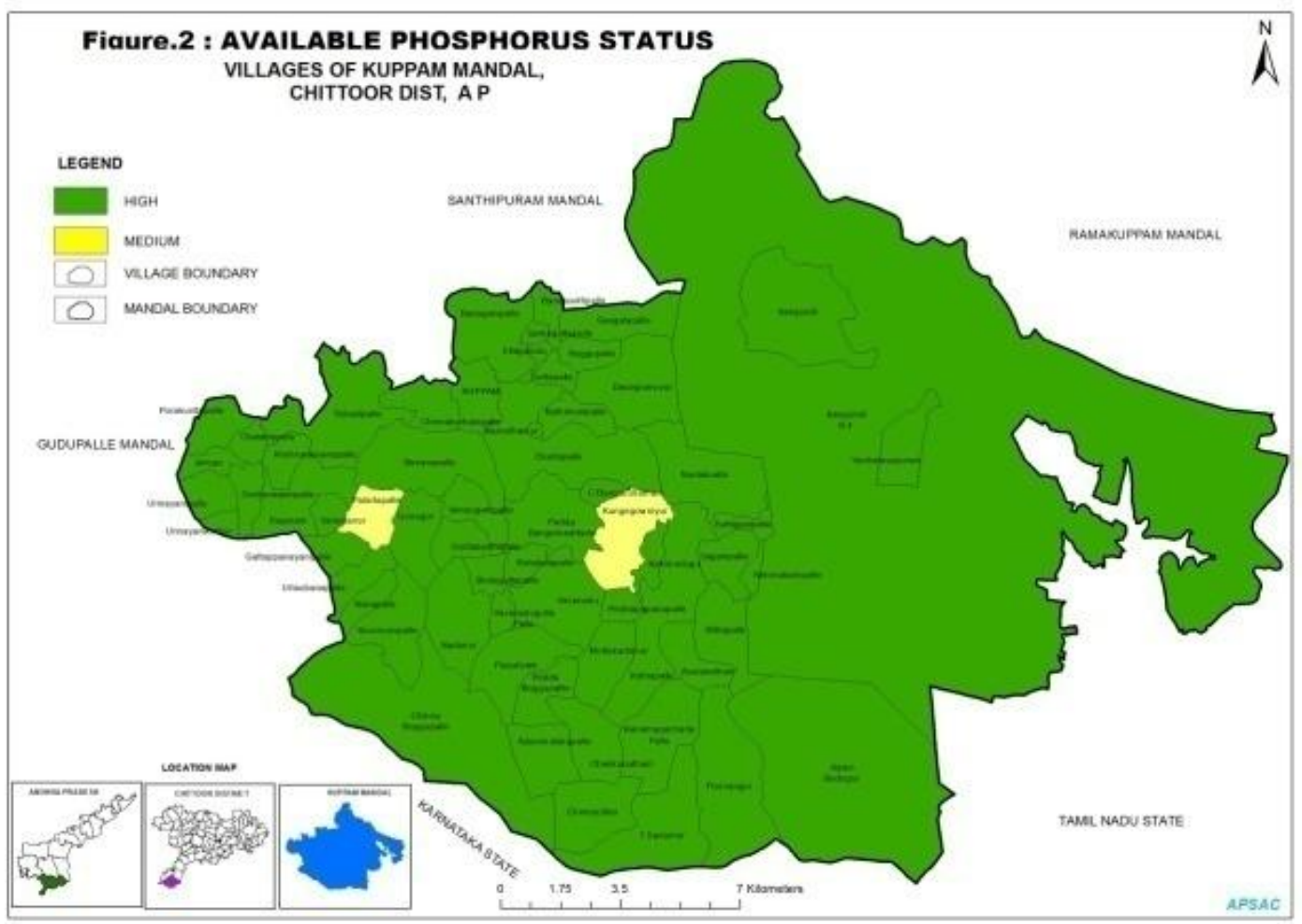


Fig.3

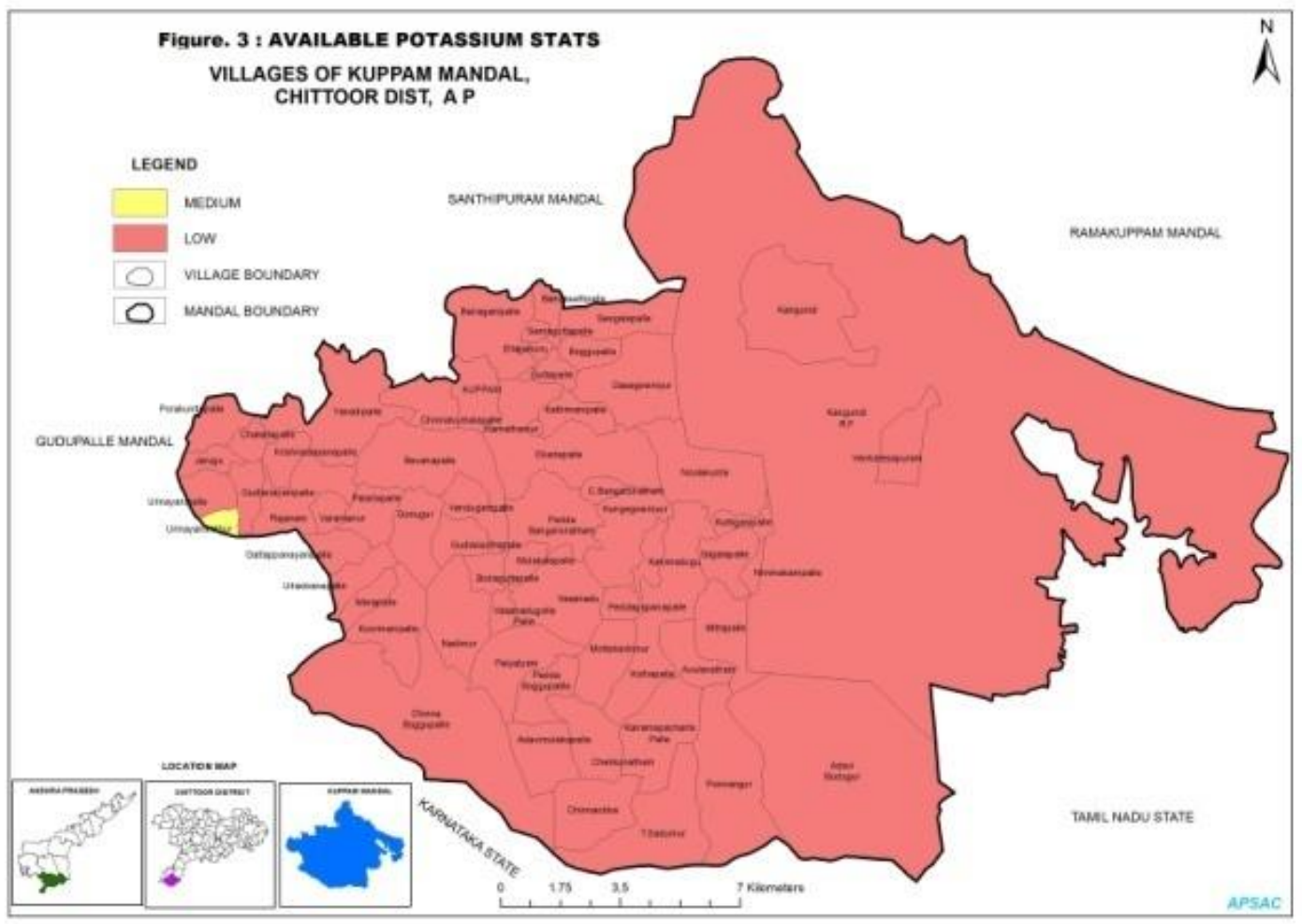

Fig.4

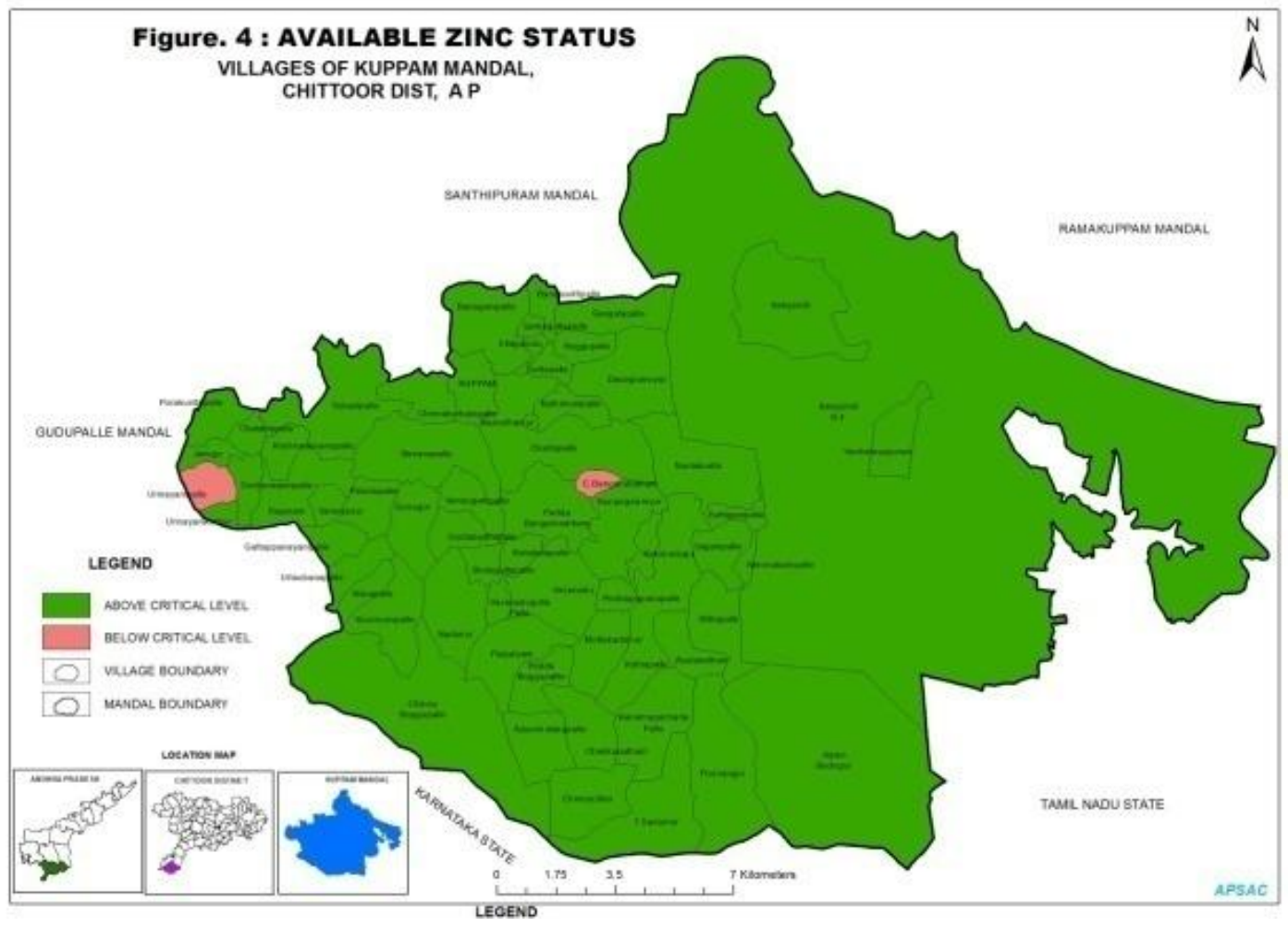


Fig.5

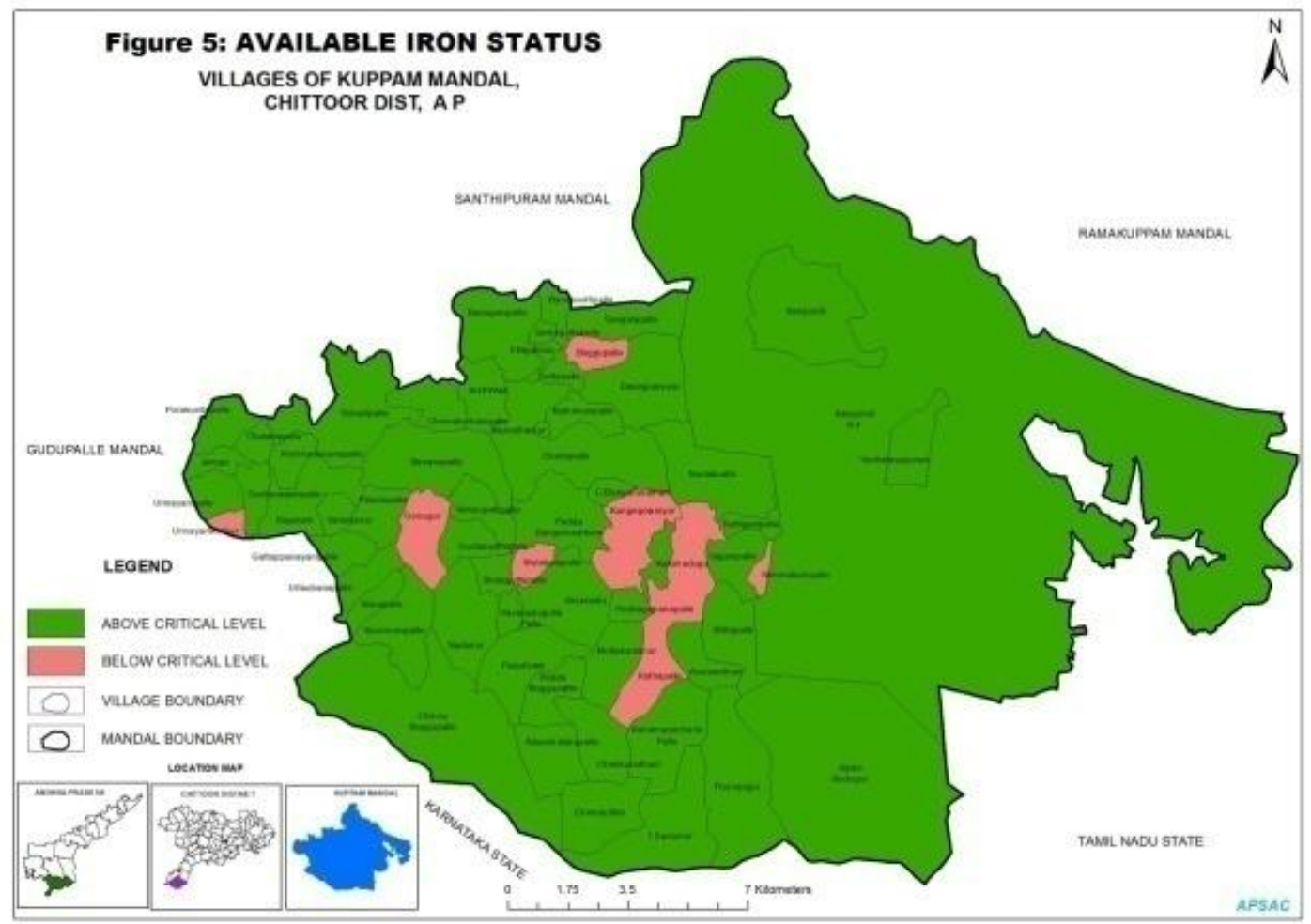

Fig.6

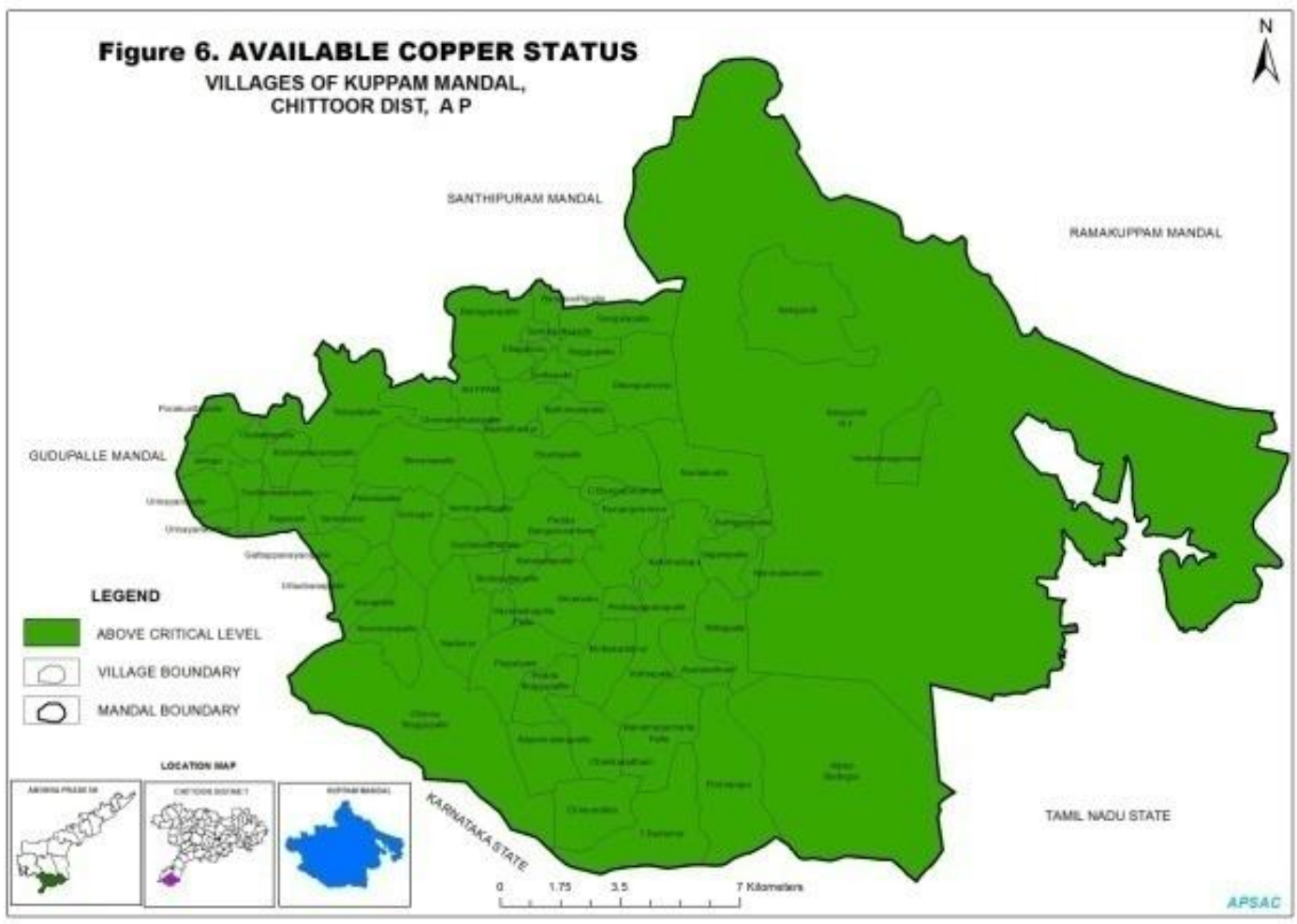


Fig.7

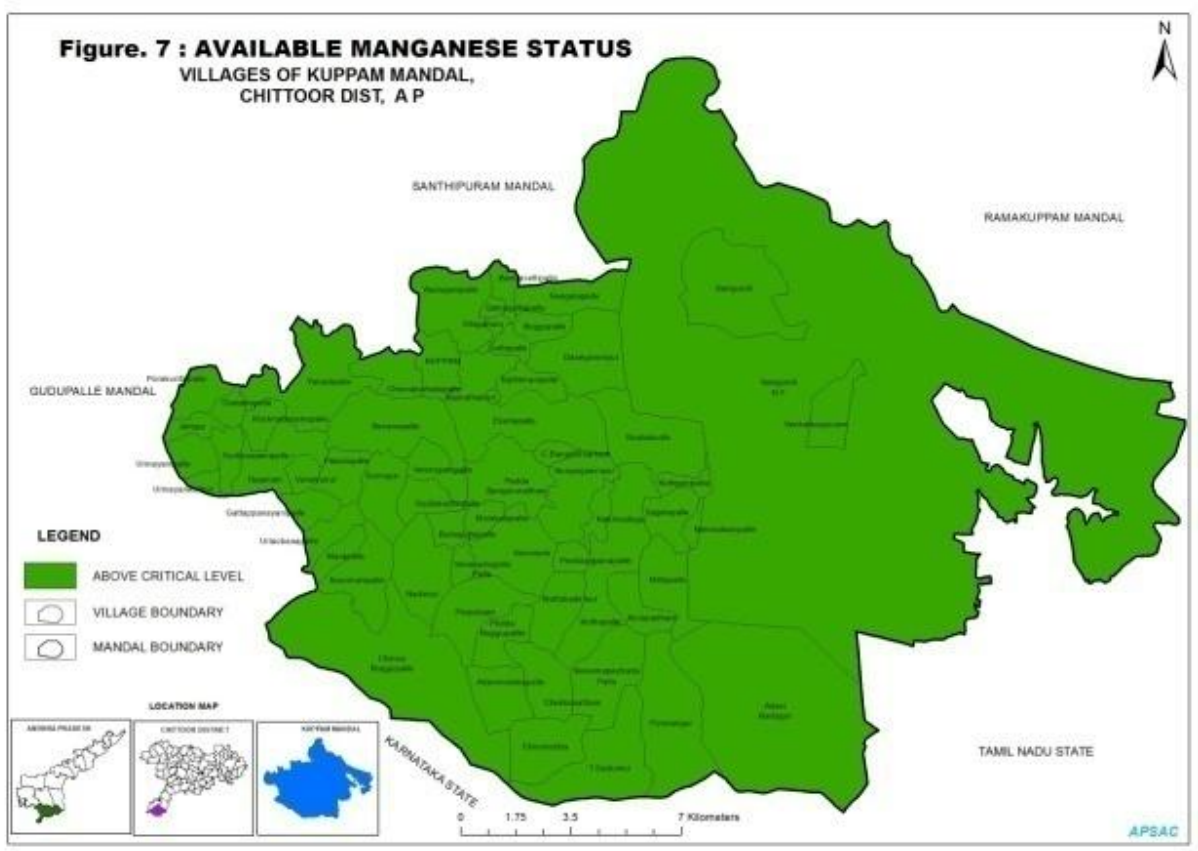

The spatial content of the available $\mathrm{K}_{2} \mathrm{O}$ is presented in Figure 3 shows that $651(47.1 \%)$ samples recorded low, 549 (39.8\%) samples recorded medium and only $181(13.1 \%)$ samples recorded high status (Table 1). Earlier we use to say that most of our soils are rich in potassium but this result shows the depletion of potassium reserves and need for balanced fertilization. As against the general perception, most of the soils in the study area are having low to medium Potassium content. So application of required quantities of potassium fertilizers based on soil test results is required to achieve the yield enhancement (Bansal, 1999).

With the intensive cropping of high yielding varieties deficiencies of Zinc ( $\mathrm{Zn}$ ) initially, and subsequently deficiencies of Iron (Fe) and Manganese emerged as threats to sustaining high levels of production (Singh 2008). The DTPA extractable nutrients were analysed and the available Zinc status was sufficient in 1089 (78.9\%) samples and $292(21.1 \%)$ (Table 2) samples recorded deficiency of Zinc (Figure 4). Available iron was sufficient in
$878(63.6 \%)$ samples and deficient in 503 (36.4\%) (Table 2) samples (Figure 5). Copper is sufficient in all most all the soil samples 1335 (96.7\%) (Table 2) and deficient in only $46(3.3 \%)$ samples (Figure 6). Manganese is also sufficient in majority $1282(92.8 \%)$ samples and deficient in only $99(7.2 \%)$ samples (Figure 7) and (Table 2).These results clearly shows that there is a scope to increase the yield by applying micronutrients also where ever needed.

In conclusion, from the study it can be concluded that most of the soils of Kuppam mandal are having very low available nitrogen and also the farmers are using less nitrogenous fertilizers than the required against the general perception of high nitrogen use. So by applying sufficient quantities of nitrogen higher yields can be achieved. The practice of phosphorus top dressing is there in this mandal also, we have to educate the farmers to avoid this, by which cost of cultivation can be reduced. Generally all our soils are rich in potassium, but due to continuous cultivation and imbalanced 
fertilization potassium deficiency is also observed. Micronutrient deficiencies were also observed though not in major area, but it is a matter of concern to achieve the double digit growth. Soil nutrient status thematic maps were generated by using Geostatistical variogram analysis and spatial interpolation (kriging). These maps will be used to make site specific fertilizer recommendations to any crop for precision agriculture to achieve sustainability.

\section{References}

Bansal, S.K. 1999. Changing pattern of crop responses to potassium in India, Paper presented at IPI-PRII-KKV Workshop on Nutrition Management in Hort. Crops, Dapoli, 11-12 Feb., 1999.

Burrough, P.A. 1993 Soil variability: A late 20th century view. Soils and fertilizers, 56: 531-562.

Cai, C.F., Ding, S.W., Zhang, G.Y., Huang, L., Wang, D.H. 1996. A preliminary study on the conditions and losses of nutrients of purple soils in Three-Gorge Reservoir area. Geography Res., 15(3): 77-84.

Cann, C. 1994. Factors of transfer of nitrate to water and their ways of action. $J$. European Hydrol., 25: 153-167.

Chen, X., Jiang, S.Q., Zhang, K.Z., Bian, Z.P. 1999. Law of phosphorus loss and its affecting factors in red soil slope land. $J$. Soil Erosion and Soil Water Conservation, 5(3): 38-41.

Jackson, M.L. 1973. Soil Chemical Analysis. Oxford, IBH Publishing house, Bombay, PP: 38
Lindsay, W.L and Norwell, W.A. 1978. Development of DTPA soil test for zinc, iron, manganese and copper. Soil Sci. America J., 43: 421-428.

Malek Mohd Yusoff, Kamaruzaman Jusoff and Mohd. Hasmadi Ismail. 2007. Soil nutrient variability mapping in UiTM research station, Arau, Perlis Using Landsat TM7 and geostatistical analysis, WSES Transaction on Signal Processing. Greece. Issue 1, Volume 3, January 2007. ISSN 1790-502:80-87.

Mc Cauley, J.D., Whittaker, A.D and Searcy, S.W. 1997. Sampling resolutions for prescription farming and their effects on cotton yield. Transactions of the American Society of Agri. Engineers.

Olsen, S.R and Watanabe, F.S. 1965. Test of an ascorbic acid method for determining phosphorous in soils. Soil Sci. America Proceedings, 29: 674-678.

Olsen, S.R, Cole, C.V, Watanabe, F.S and Dean, L.A. 1954. Estimation of available phosphorus in soils by extraction with sodium bicarbonate. Circular of United States Department of Agriculture. Pp. 939.

Singh, M.V. 2008. Chapter 4, Micronutrient deficiencies in crops and soils of India, Micronutrient deficiencies in global crop production B.J. Alloway (ed.)

Soil Survey Staff. 1997. Rating for chemical properties of soil in Peninsular Malaysia. Soil Management Division, Department of Agriculture, June, 1997.

Subbaiah, B.V and Asija, C.L. 1956. A rapid procedure for the estimation of available nitrogen in soils, Curr. Sci., 25: 32.

\section{How to cite this article:}

Reddy, P.V.R.M., K.V. Naga Madhuri, T. Giridhara Krishna and Nagarjuna, V. 2017. Mapping Soil Nutrient Content Using Geo-statistical Techniques in Kuppam Mandal of A.P. Int.J.Curr.Microbiol.App.Sci. 6(3): 852-862. doi: https://doi.org/10.20546/ijcmas.2017.603.100 